PROCEEDINGS OF THE

AMERICAN MATHEMATICAL SOCIETY

Volume 131, Number 4, Pages 1311-1320

S 0002-9939(02)06650-9

Article electronically published on September 5, 2002

\title{
PSEUDOCOMPACT TOPOLOGICAL GROUP REFINEMENTS OF MAXIMAL WEIGHT
}

\author{
W. W. COMFORT AND JORGE GALINDO
}

(Communicated by Alan Dow)

\begin{abstract}
It is known that a compact metrizable group admits no proper pseudocompact topological group refinement. The authors show, in contrast, that every (Hausdorff) pseudocompact Abelian group $G=(G, \mathcal{T})$ of uncountable weight $\alpha$, satisfying any of the following conditions, admits a pseudocompact group refinement of maximal weight (that is, of weight $2^{|G|}$ ):

(i) $G$ is compact;

(ii) $G$ is torsion-free with $\alpha \leq|G|=|G|^{\omega}$;

(iii) $[\mathrm{GCH}] G$ is torsion-free.

Remark. (i) answers a question posed by Comfort and Remus [Math. Zeitschrift 215 (1994), 337-346].
\end{abstract}

\section{INTRODUCTION}

All spaces considered here are completely regular and Hausdorff (i.e., Tychonoff spaces).

The pseudocompact spaces introduced and studied a half-century ago by Hewitt [18] are by definition those on which each real-valued continuous function is bounded; a property readily shown equivalent is that each locally finite family of open subsets is finite.

A topological group $G$ which (as a topological space) is pseudocompact is totally bounded in the sense that for each nonempty open $U \subseteq G$ there is finite $F \subseteq G$ such that $G=F U$ [10, (1.1)]. According to a non-trivial theorem of Weil [22], a topological group $G$ is totally bounded if and only if it is a topological subgroup of some compact group $K$. Clearly in this case, replacing $K$ by $\bar{G}^{K}$ if necessary, $G$ may be taken dense in $K$. The compact group $K$ containing a given totally bounded group $G$ is unique in an obvious sense 22, we denote it $\bar{G}$ and refer to it as the Weil completion of $G$.

Received by the editors August 21, 2000 and, in revised form, December 4, 2001.

2000 Mathematics Subject Classification. Primary 22A05, 54H11.

Key words and phrases. Topological group, pseudocompact, refinement topology, maximal weight.

This paper is based on work completed during the visit of the second-listed author to the Department of Mathematics of Wesleyan University, during the Fall Term of the academic year 1998-1999.

The work of the second author was supported in part by Spanish DGES, grant number BFM 2000-0913. The second author acknowledges with thanks hospitality and support received from the Department of Mathematics of Wesleyan University. 
1.1. Theorem ([10]). A topological group $G$ is pseudocompact if and only if it is (totally bounded and is) $G_{\delta}$-dense in its Weil completion $\bar{G}$ (in the sense that $G$ meets each nonempty $G_{\delta}$-subset of $\bar{G}$ ).

As a matter of convenience in pursuing his/her private thoughts, the reader may choose to follow the authors in thinking of the characterization given in Theorem 1.1 as the definition of a pseudocompact topological group.

Theorem 1.1 has two useful corollaries (cf. [10]).

1.2. Theorem. (a) The product of (any set of) pseudocompact groups is pseudocompact; and

(b) a $G_{\delta}$-dense subgroup of a pseudocompact group is pseudocompact.

Proof. The Weil completion of a totally bounded group is unique. Now in (a) the product, and in (b) the indicated subgroup, is $G_{\delta}$-dense in its Weil completion.

We say for topologies $\mathcal{T}$ and $\mathcal{V}$ on a set $X$ that $\mathcal{V}$ is a refinement of $\mathcal{T}$ if $\mathcal{T} \subseteq \mathcal{V}$; and a topology $\mathcal{V}$ is of maximal weight if $w(X, \mathcal{V})=2^{|X|}$.

There is extensive literature supporting the conjecture that every pseudocompact topological group of uncountable weight admits a proper pseudocompact topological group refinement. (The restriction on weight is necessary: An elementary argument, given for example in [7, (3.1)], shows that a pseudocompact topological group of countable weight (a) is compact metric and (b) admits no proper pseudocompact group refinement.) Concerning this conjecture, the following results from the literature are suggestive but inconclusive.

1.3. Theorem. (a) [8, (7.5)] Every pseudocompact Abelian group of uncountable weight and with a basis of clopen sets admits a proper pseudocompact group refinement.

(b) [6, (3.4)] Every compact Abelian group of uncountable weight admits a proper pseudocompact group refinement.

(c) [5. (5.5)] Every Abelian group which admits a compact group topology also admits a pseudocompact group topology of maximal weight.

The juxtaposition of parts (b) and (c) of Theorem 1.3 suggests the possibility that every compact Abelian group admits a (necessarily proper) pseudocompact group refinement of maximal weight. This question is posed explicitly by the authors of [5], who obtain a positive answer in many special cases. Our goal in this paper is to answer this question affirmatively in full generality (Theorem 5.1), and to also show that every torsion-free pseudocompact Abelian group $G$ with $\omega<w(G) \leq|G|=$ $|G|^{\omega}$ admits a pseudocompact group refinement of maximal weight (Corollary 5.3).

The technique we use to treat the case $r_{0}(G)=|G|>\mathfrak{c}$ of Theorem 5.1 is new. This paper is self-contained but where possible we invoke and adapt the technique of [6] as developed in [5] to handle the cases $r_{0}(G)<|G|$ or $|G|=\mathfrak{c}$.

1.4. Remarks. (a) The question of which Abelian groups admit a pseudocompact group topology has been examined in some detail [15], 12], [13], [14], [3], [4].

(b) Although the theorem cited above from [22] and also Theorem 1.1 place no algebraic restriction on the group $G$ or its completion, we are concerned in this paper almost exclusively with Abelian groups. This is a matter of weakness, not of choice. The questions considered in this paper admit natural unrestricted analogues, but these seem quite beyond the scope and grasp of present techniques. 
(c) Our results were presented by the second author at the 14th Summer Conference on General Topology and Its Applications (C. W. Post College, August, 1999).

\section{Notation, DEFINITIONS, AND PRELIMINARY RESUlts}

2.1. [Cardinals] The symbols, $\alpha, \beta, \gamma$ and $\kappa$ denote cardinal numbers, usually infinite; $\omega$ is the least infinite cardinal; and $\mathfrak{c}:=2^{\omega}$. The symbol $|X|$ is the cardinality of the set $X$, and $[X]^{\kappa}=\{A \subseteq X:|A|=\kappa\}$.

2.2. [Algebra] $\mathbb{Z}$ and $\mathbb{T}$ denote the group of integers and the circle group, respectively; $\mathbb{P}:=\{p \in \mathbb{Z}: p$ is prime $\}$, and $\mathbb{Z}(p)$ is the cyclic group of order $p$.

The identity element of an Abelian group $G$ is denoted 0 or $0_{G}$.

The torsion-free rank and the $p$-rank (for $p \in \mathbb{P}$ ) are denoted $r_{0}(G)$ and $r_{p}(G)$, respectively, and $r(G):=r_{0}(G)+\sum_{p \in \mathbb{P}} r_{p}(G)$. It is known that $|G|=r(G)$ when $|G|>\omega($ cf. [16, $\S 16])$.

2.3. [Topology] The weight of a topological space $X=(X, \mathcal{T})$ is denoted $w(X)$ or $w(X, \mathcal{T})$.

2.4. [Topological Groups] The group of continuous homomorphisms from a topological Abelian group to the group $\mathbb{T}$ is denoted $\widehat{G}$. It is well-known (see for example [19]) that a compact Abelian group $G$ with $w(G)=\alpha \geq \omega$ satisfies $|G|=2^{\alpha}$ and $|\widehat{G}|=\alpha$.

2.5. For each topological group $G$ we write

$$
\Lambda(G)=\left\{N \subseteq G: N \text { is a closed, normal } G_{\delta} \text {-subgroup of } G\right\} .
$$

According to the Kakutani-Kodaira theorem (as exposed, for example, in [19. (8.7)]), every nonempty $G_{\delta}$-set containing the identity of a group $G$ also contains an element of $\Lambda(G)$. The following facts are basic to our investigation.

2.6. Theorem. Let $G$ be an infinite pseudocompact group. Then:

(a) $|G| \geq \mathfrak{c}$;

(b) each $N \in \Lambda(G)$ is pseudocompact;

(c) (for $G$ Abelian) either $G$ is a torsion group or $r_{0}(G) \geq \mathfrak{c}$; and

(d) (for $G$ Abelian) if $r_{0}(G)>\mathfrak{c}$ and $N \in \Lambda(G)$, then $r_{0}(N)=r_{0}(G)$.

Proof. With no claim for priority we refer the reader to [7, (2.5)] for (a) and to [8, (6.2)] for (b). The proof in [5] (4.1)] of the compact case of (c) readily adapts here; see also [11, (2.17)] for the general statement. In (d) the group $G / N$ is compact metric, so $r_{0}(G / N) \leq|G / N| \leq \mathfrak{c}$; then $r_{0}(N)=r_{0}(G)$ follows from $r_{0}(G)=r_{0}(G / N)+r_{0}(N)$.

2.7. Discussion. It is shown in [7 that for $\alpha \geq \omega$ and $K$ a compact group with $w(K)=\alpha$, the cardinal number

$$
\min \{|G|: G \text { is a dense pseudocompact subgroup of } K\}
$$

depends only on $\alpha$ (and not on any algebraic properties of $K$ ). Here we follow 7] and denote the number $\left(^{*}\right)$ by the symbol $\mathrm{m}(\alpha)$. (See [1] and 14] for other notations.) It is clear from Theorem 2.6(a) that every $\alpha \geq \omega$ satisfies $\mathrm{m}(\alpha) \geq \mathfrak{c}$. The following additional inequalities, verified in [7] and elsewhere, will be used in $\S 5$. 
2.8. Theorem. Let $\alpha \geq \omega$. Then:

(a) $\operatorname{cf}(\mathrm{m}(\alpha))>\omega$ and

(b) $\log (\alpha) \leq \mathrm{m}(\alpha) \leq(\log (\alpha))^{\omega}$.

\section{Compact Abelian groups: When $|G|=\mathfrak{c}$ or $r_{0}(G)<|G|$}

In this section (only), given a compact Abelian group $G$ with $w(G)=\alpha>\omega$, we reserve the symbols $\alpha_{0}$ and $\alpha_{p}(p \in \mathbb{P})$ to denote $r_{0}(\widehat{G})$ and $r_{p}(\widehat{G})$, respectively.

For proofs of the following two results the reader may consult [5] (5.4) and [5] (5.2), respectively.

3.1. Theorem. Let $G$ be a compact Abelian group with $w(G)=\alpha>\omega$. Then there is a continuous surjective homomorphism $h: G \rightarrow \mathbb{T}^{\alpha_{0}} \times \prod_{p \in \mathbb{P}}(\mathbb{Z}(p))^{\alpha_{p}}$.

3.2. Theorem. Let $(G, \mathcal{T})$ and $K$ be compact groups with $|K|>1$ and $K$ metrizable. If there is a continuous surjective homomorphism $h: G \rightarrow K^{\kappa}$ with $\kappa>\omega$, then there is a pseudocompact group topology $\mathcal{V}$ on $G$ such that $\mathcal{V} \supseteq \mathcal{T}, \mathcal{V} \neq \mathcal{T}$, and $w(G, \mathcal{V})=w(G, \mathcal{T})+2^{2^{\kappa}}$.

3.3. Corollary. Let $G=(G, \mathcal{T})$ be a compact Abelian group with $w(G)=\alpha>\omega$. If $|G|=\mathfrak{c}$, then there is a pseudocompact group topology $\mathcal{V}$ for $G$ such that $\mathcal{V} \supseteq \mathcal{T}$, $\mathcal{V} \neq \mathcal{T}$, and $w(G, \mathcal{V})=2^{|G|}$.

Proof. Since $\omega<\alpha=|\widehat{G}|=\alpha_{0}+\Sigma_{p \in \mathbb{P}} \alpha_{p}$, some $\kappa \in\left\{\alpha_{0}\right\} \cup\left\{\alpha_{p}: p \in \mathbb{P}\right\}$ satisfies $\omega<\kappa \leq \alpha<2^{\alpha}=\mathfrak{c}$ and hence $2^{\kappa}=\mathfrak{c}$. By Theorem 3.1 there is a continuous homomorphism from $(G, \mathcal{T})$ onto either $\mathbb{T}^{\kappa}$ or $(\mathbb{Z}(p))^{\kappa}$, so Theorem 3.2 applies.

Next we achieve the conclusion of Corollary 3.3 for compact Abelian groups $G$ with $r_{0}(G)<|G|$. Our proof of the following lemma parallels that of 5 , (5.5(c))] (cf. also [20, proof of 4.10]).

3.4. Lemma. Let $G$ be a compact Abelian group with $w(G)=\alpha>\omega$. Then either $r_{0}(G)=|G|$ or there is $p \in \mathbb{P}$ such that $|G|=2^{\alpha_{p}}$.

Proof. By Theorem 3.1 there is a continuous homomorphism from $G$ onto the group $H:=\mathbb{T}^{\alpha_{0}} \times \prod_{p \in \mathbb{P}}(\mathbb{Z}(p))^{\alpha_{p}}$. Since $w(H)=\alpha_{0}+\sum_{p \in \mathbb{P}} \alpha_{p}=\alpha$ we have $2^{\alpha}=|H|=$ $r(H)=r_{0}(H)+\sum_{p \in \mathbb{P}} r_{p}(H)$ by subsections 2.4 and 2.2 (applied to $H$ ). Since $\operatorname{cf}\left(2^{\alpha}\right)>\omega$, either $r_{0}(H)=2^{\alpha}$ or $r_{p}(H)=2^{\alpha}$ for some $p \in \mathbb{P}$. If $r_{0}(H)=2^{\alpha}$, then $2^{\alpha}=|G| \geq r_{0}(G) \geq r_{0}(H)=2^{\alpha}$ so $r_{0}(G)=|G| ;$ and if $r_{0}(H)<2^{\alpha}=r_{p}(H)$, then $|G|=2^{\alpha}=2^{\alpha_{p}}$ follows from $2^{\alpha_{0}}=r_{0}\left(\mathbb{T}^{\alpha_{0}}\right) \leq r_{0}(H)<2^{\alpha}$ and $|G|=2^{\alpha}=r_{p}(H)=$ $r_{p}\left(\mathbb{T}^{\alpha_{0}}\right) \cdot r_{p}\left(\mathbb{Z}(p)^{\alpha_{p}}\right)=2^{\alpha_{0}} \cdot 2^{\alpha_{p}}$.

3.5. Theorem. Let $G=(G, \mathcal{T})$ be a compact Abelian group with $w(G)=\alpha>\omega$. If $r_{0}(G)<|G|$, then there is a pseudocompact group topology $\mathcal{V}$ for $G$ such that $\mathcal{V} \supseteq \mathcal{T}, \mathcal{V} \neq \mathcal{T}$ and $w(G, \mathcal{V})=2^{|G|}$

Proof. By Theorem 3.4 there is $p \in \mathbb{P}$ such that $|G|=2^{\alpha_{p}}$, and by Lemma 3.1 there is a continuous homomorphism from $G$ onto $(\mathbb{Z}(p))^{\alpha_{p}}$. The statement then follows from Theorem 3.2 (with $\kappa=\alpha_{p}$ ). 


\section{Compact Abelian groups: When $r_{0}(G)=|G|>\mathfrak{c}$}

4.1. Lemma. Let $G=(G, \mathcal{T})$ and $H$ be pseudocompact topological groups with $w(G)=\alpha>\omega$ and $w(H)=\kappa \geq \omega$. If there is a homomorphism $h$ from $G$ into $H$ such that each $N \in \Lambda(G)$ has $h[N] G_{\delta}$-dense in $H$, then there is a pseudocompact group topology $\mathcal{V}$ for $G$ such that $\mathcal{V} \supseteq \mathcal{T}, \mathcal{V} \neq \mathcal{T}$ and $w(G, \mathcal{V})=\alpha+\kappa$.

Proof. The set $\operatorname{graph}(h)$ is a $G_{\delta}$-dense subgroup of $G \times H$. (For, let $a N \times V$ be a "basic $G_{\delta}$-set" in $G \times H$. Here $a \in G, N \in \Lambda(G)$, and $V$ is a $G_{\delta}$-subset of $H$. By hypothesis there is $t \in N$ such that $h(t) \in h\left(a^{-1}\right) V$, and then $(a t, h(a t)) \in$ $\operatorname{graph}(h) \cap(a N \times V)$.) The group $\operatorname{graph}(h)$ is then pseudocompact by Theorem 1.2(b), so $G$ is pseudocompact in the topology $\mathcal{V}$ defined by the requirement that the map $x \rightarrow(x, h(x))$ from $(G, \mathcal{V})$ onto graph $(h)$ is a homeomorphism. Since weight is invariant under passage from a topological group to a dense subgroup, we have

$$
w(G, \mathcal{V})=w(\operatorname{graph}(h))=w((G, \mathcal{T}) \times H) ;
$$

the conditions $\mathcal{V} \supseteq \mathcal{T}$ and $\mathcal{V} \neq \mathcal{T}$ are easily checked.

4.2. Remark. Although the product of pseudocompact topological groups is pseudocompact [10, (1.4)], it is not difficult to produce Abelian groups $G$ with pseudocompact group topologies $\mathcal{T}_{0}$ and $\mathcal{T}_{1}$ such that the supremum $\mathcal{T}_{0} \vee \mathcal{T}_{1}$, though necessarily a group topology for $G$, is not pseudocompact. (For an example to this effect let $h$ be a discontinuous automorphism of any compact metric group $\left(G, \mathcal{T}_{0}\right)$ and let $\mathcal{T}_{1}$ be the topology defined on $G$ by the requirement that $h:\left(G, \mathcal{T}_{0}\right) \rightarrow\left(G, \mathcal{T}_{1}\right)$ is a homeomorphism. As noted earlier, no pseudocompact group topology on $G$ properly contains $\mathcal{T}_{0}$; in particular, $\mathcal{T}_{0} \vee \mathcal{T}_{1}$ is not pseudocompact.) Accordingly it is useful to notice that the proof of Lemma 4.1 gives a simple and workable criterion under which such a supremum topology will indeed be pseudocompact, as follows.

Let $\mathcal{T}_{0}$ and $\mathcal{T}_{1}$ be pseudocompact group topologies on a group $G$ such that each $N \in \Lambda\left(G, \mathcal{T}_{0}\right)$ is $G_{\delta}$-dense in $\left(G, \mathcal{T}_{1}\right)$. Then $\mathcal{T}_{0} \vee \mathcal{T}_{1}$ is a pseudocompact group topology on $G$.

To prove this statement it is enough to consider the homomorphism $h: G=$ $\left(G, \mathcal{T}_{0}\right) \rightarrow H=\left(G, \mathcal{T}_{1}\right)$ given by $h(x)=x$. Since $\operatorname{graph}(h)$ is the "diagonal" copy of $G$ in $\left(G, \mathcal{T}_{0}\right) \times\left(G, \mathcal{T}_{1}\right)$, the pseudocompact topology $\mathcal{V}$ defined in the proof of Lemma 4.1 is $\mathcal{T}_{0} \vee \mathcal{T}_{1}$.

The perspective afforded by the foregoing observation is useful in addressing the question of the existence of pseudocompact topological groups $(G, \mathcal{T})$ of uncountable weight which are extremal in the sense that either $G$ contains no proper dense pseudocompact subgroup or $\mathcal{T}$ admits no proper pseudocompact topological group refinement [2].

The next two lemmas, of combinatorial flavor, pave the way for the construction of proper pseudocompact group refinements of a pseudocompact $\operatorname{group}(G, \mathcal{T})$ in the case that $r_{0}(G)$ is large.

As usual, we say that a subset $X$ of an Abelian group $G$ is independent if each $x \in X$ satisfies $\langle x\rangle \cap\langle X \backslash\{x\}\rangle=\left\{0_{G}\right\}$.

4.3. Lemma. Let $H$ be a subgroup of an Abelian group $G$ and let $X$ be an independent subset of $G$ such that $|H|<|X|$. Then there is $x \in X$ such that $\langle x\rangle \cap H=\left\{0_{G}\right\}$. 
Proof. If the statement fails, then for $x \in X$ there is $n_{x} \in \mathbb{Z}$ such that $0_{G} \neq n_{x} \cdot x \in$ $H$. The map $X \rightarrow H$ given by $x \rightarrow n_{x} \cdot x$ is not one-to-one, so there are distinct $x, y \in X$ such that

$$
0_{G} \neq n_{x} \cdot x=n_{y} \cdot y \in\langle x\rangle \cap\langle y\rangle \subseteq\langle x\rangle \cap\langle X \backslash\{x\}\rangle=\left\{0_{G}\right\},
$$

a contradiction.

4.4. Lemma. Let $\beta \geq \omega$, let $G$ be an Abelian group, and for $\eta<\beta$ let $X_{\eta} \in[G]^{\beta}$ be an independent set of non-torsion elements. Then there is a family $\left\{B_{\eta}: \eta<\right.$ $\beta\} \subseteq \mathcal{P}(G)$ such that

(i) $B_{\eta} \in\left[X_{\eta}\right]^{\beta}$ for $\eta<\beta$,

(ii) $B_{\eta^{\prime}} \cap B_{\eta}=\emptyset$ for $\eta^{\prime}<\eta<\beta$, and

(iii) $\bigcup_{\eta<\beta} B_{\eta}$ is an independent set.

Proof. Let $S:=\{(\delta, \eta): \eta \leq \delta<\beta\}$ be lexicographically ordered in the usual way: $\left(\delta^{\prime}, \eta^{\prime}\right)<(\delta, \eta)$ if either (a) $\delta^{\prime}<\delta$ or (b) $\delta^{\prime}=\delta$ and $\eta^{\prime}<\eta$. We will use transfinite induction over $S$ to choose $x_{\delta, \eta} \in X_{\eta}$ so that

$$
\left\langle x_{\delta, \eta}\right\rangle \cap\left\langle\left\{x_{\delta^{\prime}, \eta^{\prime}}:\left(\delta^{\prime}, \eta^{\prime}\right)<(\delta, \eta)\right\}\right\rangle=\{0\} \quad \text { for }(\delta, \eta) \in S .
$$

To begin, choose $x_{0,0} \in X_{0}$. Now let $(\rho, \sigma) \in S$, suppose for all $(\delta, \eta)<(\rho, \sigma)$ that $x_{\delta, \eta}$ has been chosen in $X_{\eta}$ so that $\left(^{*}\right)$ holds, and let $H_{\rho, \sigma}=\left\langle\left\{x_{\delta, \eta}:(\delta, \eta)<(\rho, \sigma)\right\}\right\rangle$. Since $\left|H_{\rho, \sigma}\right|<\beta=\left|X_{\sigma}\right|$, by Lemma 4.3 there is $x_{\rho, \sigma} \in X_{\sigma}$ such that $\left\langle x_{\rho, \sigma}\right\rangle \cap H_{\rho, \sigma}=$ $\{0\}$, i.e., $(*)$ holds for $(\delta, \eta)=(\rho, \sigma)$. The inductive construction is complete, $\left(^{*}\right)$ holds for all $(\delta, \eta) \in S$, and it is enough to set $B_{\eta}:=\left\{x_{\delta, \eta}: \eta \leq \delta<\beta\right\}$.

4.5. Theorem. Let $G=(G, \mathcal{T})$ be a pseudocompact Abelian group with $w(G)=$ $\alpha>\omega$, and set $\beta=\min \left\{r_{0}(N): N \in \Lambda(G)\right\}$. If $\alpha^{\omega} \leq \beta$ and if $\kappa \geq \omega$ satisfies $\mathrm{m}(\kappa) \leq \beta$, then $G$ admits a pseudocompact group topology $\mathcal{V}$ such that $\mathcal{V} \supseteq \mathcal{T}$, $\mathcal{V} \neq \mathcal{T}$, and $w(G, \mathcal{V})=\alpha+\kappa$.

Proof. Let $A$ be a dense pseudocompact subgroup of $\mathbb{T}^{\kappa}$ such that $|A|=\mathrm{m}(\kappa)$.

Since $w(G)=\alpha \leq \alpha^{\omega} \leq \beta$ there is a (not necessarily faithfully indexed) set $\left\{N_{\eta}: \eta<\beta\right\} \subseteq \Lambda(G)$ such that each $N \in \Lambda(G)$ contains some $N_{\eta}$. Since each $r_{0}\left(N_{\eta}\right) \geq \beta$, there is an independent set $X_{\eta} \in\left[N_{\eta}\right]^{\beta}$ of non-torsion elements; then by Lemma 4.4 there is $B_{\eta} \in\left[X_{\eta}\right]^{\beta}$ such that $B:=\bigcup_{\eta<\beta} B_{\eta}$ is independent. Let $h: B \rightarrow A$ be any function such that $h\left[B_{\eta}\right]=A$ for each $\eta<\beta$, and extend $h$ first to a homomorphism from $\langle B\rangle$ onto $\langle A\rangle=A$ and then to a homomorphism (also denoted $h$ ) from $G$ into the divisible group $\mathbb{T}^{\kappa}$. Since each $N \in \Lambda(G)$ has $h[N] \supseteq h\left[N_{\eta}\right]=A$ for some $\eta<\beta$, the existence of $\mathcal{V}$ as required is immediate from Lemma 4.1 (with $H=\mathbb{T}^{\kappa}$ ).

4.6. Remarks. (a) If in Lemma 4.1 or Theorem 4.5 the hypothesis $w(G)=\alpha>\omega$ is replaced by $w(G)=\alpha=\omega$, the remaining hypotheses cannot be satisfied (since in this case the trivial group $\left\{0_{G}\right\} \in \Lambda(G)$ ). As noted parenthetically following Theorem 1.2, the conclusions of Lemma 4.1 and Theorem 4.5 also fail in this case.

(b) The hypothesis $r_{0}(N) \geq \mathrm{m}(\kappa)$ of Theorem 4.5, equivalent to $r_{0}(G) \geq \mathrm{m}(\kappa)$ when $r_{0}(G)=|G|>\mathfrak{c}$, may strike the reader as unexpectedly and unnecessarily strong. The condition cannot in general be weakened, however, since (for example) it is known (cf. [3] (4.15)], or [14, (7.1)]) that every Abelian group $G$ which admits a pseudocompact connected group topology of weight $\kappa \geq \omega$ satisfies not only the obvious condition $|G| \geq \mathrm{m}(\kappa)$ but also $r_{0}(G) \geq \mathrm{m}(\kappa)$. 
(c) It is tempting to believe that the hypothesis $r_{0}(N) \geq \mathrm{m}(\kappa)$ of Theorem 4.5, which allowed a homomorphism from $(G, \mathcal{T})$ onto a $G_{\delta}$-dense subgroup of $\mathbb{T}^{\kappa}$ (and then a proper pseudocompact group refinement $\mathcal{V}$ of $\mathcal{T}$ such that $w(G, \mathcal{V}) \geq$ $\left.w\left(\mathbb{T}^{\kappa}\right)=\kappa\right)$, might be replaced by $r_{p}(N) \geq \mathrm{m}(\kappa)$ and then by a homomorphism of $(G, \mathcal{T})$ onto a $G_{\delta}$-dense subgroup of $(\mathbb{Z}(p))^{\kappa}$ (and hence, again, a pseudocompact refinement $\mathcal{V}$ of $\mathcal{T}$ such that $\left.w(G, \mathcal{V}) \geq w\left((\mathbb{Z}(p))^{\kappa}\right)=\kappa\right)$. To see that this attempt to strengthen Theorem 4.5 fails, suppose that $G$ is a divisible Abelian group. Any pseudocompact group topology $\mathcal{V}$ on $G$ (say of weight $\kappa$ ) then has a divisible completion $\overline{(G, \mathcal{V})}$ which is necessarily connected [21], and $r_{0}(G) \geq \mathrm{m}(\kappa)$ then follows from $w(G, \mathcal{V})=w(\overline{(G, \mathcal{V})})=\kappa$ and the argument of (b) above. Evidently, then, no "largeness" condition on the numbers $|G|, r_{p}(G)$ or $r_{p}(N)(N \in \Lambda(G))$ can substitute in Theorem 4.5 for the assumption $r_{0}(N) \geq \mathrm{m}(\kappa)$.

4.7. Corollary. Let $G=(G, \mathcal{T})$ be a pseudocompact Abelian group with $w(G)=$ $\alpha>\omega$ such that

(i) each $N \in \Lambda(G)$ satisfies $r_{0}(N) \geq \mathrm{m}\left(2^{|G|}\right)$, and

(ii) $r_{0}(G) \geq \alpha^{\omega}$.

Then there is a pseudocompact group topology $\mathcal{V}$ on $G$ such that $\mathcal{V} \supseteq \mathcal{T}, \mathcal{V} \neq \mathcal{T}$, and $w(G, \mathcal{V})=2^{|G|}$.

Proof. With $\kappa:=2^{|G|}$ we have $\kappa \geq \alpha$ and hence $\alpha+\kappa=\kappa$, so in order to apply Theorem 4.5 it suffices to show $r_{0}(N) \geq \alpha^{\omega}$ for each $N \in \Lambda(G)$. By (i) surely $r_{0}(N)>0$, so $r_{0}(N) \geq \mathfrak{c}$ by Lemma 2.6(a)(b) and hence $r_{0}(N) \geq \alpha^{\omega}$ if $\alpha^{\omega}=\mathfrak{c}$; and if $\alpha^{\omega}>\mathfrak{c}$, then $r_{0}(N)=r_{0}(G) \geq \alpha^{\omega}$ by Lemma 2.6(d) and (ii).

Our goal is to prove Theorem 5.1- that every compact Abelian group of uncountable weight admits a (necessarily proper) pseudocompact group refinement of maximal weight. Our principal consequence of Theorem 4.5 (indeed of Corollary 4.7 ) is the following, which allows us to deal with the special cases not handled in $\S 3$.

4.8. Corollary. Let $G=(G, \mathcal{T})$ be a compact Abelian group with $w(G)=\alpha>\omega$ such that $r_{0}(G)=|G|>\mathfrak{c}$. Then there is a pseudocompact group topology $\mathcal{V}$ on $G$ such that $\mathcal{V} \supseteq \mathcal{T}, \mathcal{V} \neq \mathcal{T}$, and $w(G, \mathcal{V})=2^{|G|}$.

Proof. From Theorems 2.8(b) and 2.6(d), for $N \in \Lambda(G)$ we have

$$
\mathrm{m}\left(2^{|G|}\right) \leq\left(\log \left(2^{|G|}\right)\right)^{\omega} \leq|G|^{\omega}=\left(2^{\alpha}\right)^{\omega}=2^{\alpha}=|G|=r_{0}(G)=r_{0}(N) ;
$$

in particular $r_{0}(G)=2^{\alpha} \geq \alpha^{\omega}$. Thus Corollary 4.7 applies.

\section{Pseudocompact Refinements of maximal Weight}

5.1. Theorem. Let $G=(G, \mathcal{T})$ be a compact Abelian group with $w(G)=\alpha>\omega$. Then there is a pseudocompact group topology $\mathcal{V}$ on $G$ such that $\mathcal{V} \supseteq \mathcal{T}, \mathcal{V} \neq \mathcal{T}$, and $w(G, \mathcal{V})=2^{|G|}$.

Proof. The cases $|G|=\mathfrak{c}$ or $r_{0}(G)<|G|$ are handled by Corollary 3.3 and Theorem 3.5, respectively, and the remaining case by Corollary 4.8.

5.2. Corollary. Let $G=(G, \mathcal{T})$ be a compact Abelian group with $w(G, \mathcal{T})=\alpha>$ $\omega$. Then with $\kappa:=2^{2^{2^{\alpha}}}$ there are $\kappa$-many pseudocompact group topologies $\mathcal{V}_{\xi}(\xi<\kappa)$ on $G$ such that $\mathcal{V}_{\xi} \supseteq \mathcal{T}, \mathcal{V}_{\xi} \neq \mathcal{T}$, and $w\left(G, \mathcal{V}_{\xi}\right)=2^{|G|}$. 
Proof. We have remarked already (Theorem 1.1) that every pseudocompact topological group is totally bounded. It is known further ([9]) that every totally bounded group topology on the Abelian group $G$ is the topology $\mathcal{T}_{A}$ induced by a suitably chosen point-separating subgroup $A$ of $\operatorname{Hom}(G, \mathbb{T})$; further, different such groups induce different topologies, and $w\left(G, \mathcal{T}_{A}\right)=|A|$.

Now let $G, \mathcal{T}$ and $\mathcal{V}$ be as in Theorem 5.1 and let $A$ and $B$ be those subgroups of $\operatorname{Hom}\left(G, \mathbb{T}\right.$ ) such that $\mathcal{T}=\mathcal{T}_{A}$ and $\mathcal{V}=\mathcal{T}_{B}$ (with necessarily $A \subseteq B,|A|=\alpha$, and $\left.|B|=w(G, \mathcal{V})=2^{|G|}=2^{2^{\alpha}}\right)$. Since $|B / A|=2^{2^{\alpha}}$ there are $\kappa$-many subgroups $C_{\xi}(\xi<\kappa)$ of $B$ such that $C_{\xi} \supseteq A$ and $\left|C_{\xi}\right|=2^{2^{\alpha}}$. With $\mathcal{V}_{\xi}:=\mathcal{T}_{C_{\xi}}(\xi<\kappa)$ the family $\left\{\mathcal{V}_{\xi}: \xi<\kappa\right\}$ is as required.

We continue with two additional miscellaneous corollaries of Theorem 4.5.

5.3. Corollary. Let $G=(G, \mathcal{T})$ be a torsion-free pseudocompact Abelian group such that $\omega<\alpha=w(G) \leq|G|=|G|^{\omega}$. Then there is a pseudocompact group topology $\mathcal{V}$ on $G$ such that $\mathcal{V} \supseteq \mathcal{T}, \mathcal{V} \neq \mathcal{T}$, and $w(G, \mathcal{V})=2^{|G|}$.

Proof. Noticing m $\left(2^{|G|}\right) \leq\left(\log \left(2^{|G|}\right)\right)^{\omega} \leq|G|^{\omega}=|G|$ by Theorem 2.8(b), we verify hypotheses (i) and (ii) of Corollary 4.7.

(i) Since $w(G)=\alpha>\omega$, every $N \in \Lambda(G)$ satisfies $|N|>1$ and hence $r_{0}(N)=$ $|N| \geq \mathfrak{c}$. Now if $|G|=\mathfrak{c}$, then $r_{0}(N)=\mathfrak{c}=\mathrm{m}\left(2^{|G|}\right)$, and if $|G|>\mathfrak{c}$, then $r_{0}(N)=$ $r_{0}(G)=r(G)=|G| \geq \mathrm{m}\left(2^{|G|}\right)$ by Theorem $2.6(\mathrm{~d})$.

(ii) From $|G|^{\omega}=|G| \geq \alpha$ follows $|G| \geq \alpha^{\omega}$.

5.4. Corollary $(\mathrm{GCH})$. Let $G=(G, \mathcal{T})$ be a torsion-free pseudocompact Abelian group with $w(G)=\alpha>\omega$. Then

(a) either (i) $\alpha=2^{|G|}$ or (ii) $\alpha \leq|G|=|G|^{\omega}$, and

(b) there is a pseudocompact group topology $\mathcal{V}$ on $G$ such that $\mathcal{V} \supseteq \mathcal{T}$ and $w(G, \mathcal{V})=2^{|G|}$.

Proof. (a) Surely $|G| \leq 2^{\alpha}$ and $\alpha \leq 2^{|G|}$, so from GCH we have either $\alpha=2^{|G|}$ (which is (i)) or $|G|=2^{\alpha}$ (hence (ii)) or $|G|=\alpha$. Now if $|G|=\alpha$ and (ii) fails, then $\alpha=|G|<|G|^{\omega}$, so $\operatorname{cf}(\alpha)=\omega$ and $\alpha=\log (\alpha)$; then from GCH and Theorem 2.8(a) $\mathrm{m}(\alpha)=(\mathrm{m}(\alpha))^{\omega}$ follows, so

$$
2^{\alpha}=\alpha^{\omega}=(\log (\alpha))^{\omega}=(\mathrm{m}(\alpha))^{\omega}=\mathrm{m}(\alpha) \leq|G| \leq 2^{\alpha}
$$

and hence (ii) holds.

(b) If (a)(i) holds, take $\mathcal{V}=\mathcal{T}$, and if (a)(ii) holds, apply Corollary 5.3.

\section{Concluding COMments And A QUESTION}

6.1. Remark. It is reasonable to inquire whether every compact Abelian group group $G$, say with $w(G)=\alpha>\omega$, maps by a continuous homomorphism onto some (compact) group of the form $F^{\kappa}$ with $|F|>1$ and with $2^{2^{\kappa}}=2^{2^{\alpha}}$. (For if so, then $F$ may be chosen metrizable and Theorem 3.2 above cited from [5. (5.2)] proves Theorem 5.1 immediately, and the more delicate argument presented in this paper is unnecessary.) We now show, using a group $G$ suggested in this context in [5. (5.7)] and answering the question posed there, that the answer to this question is "No". Thus the technique of [5, (5.2)] cannot apply to certain compact Abelian groups $G$. 
6.2. Theorem. Let $\alpha_{0} \geq \omega$, recursively define $\alpha_{n+1}=2^{\alpha_{n}}$, let $\left\{p_{n}: n<\omega\right\}$ faithfully enumerate $\mathbb{P}$, and set $G=\prod_{n<\omega}\left(\mathbb{Z}\left(p_{n}\right)\right)^{\alpha_{n}}$ (with the usual compact topology). Then $w(G)=\alpha:=\sum_{n<\omega} \alpha_{n}$, and if $h: G \rightarrow F^{\kappa}$ is a continuous surjective homomorphism with $|F|>1$, then $2^{2^{\kappa}}<2^{2^{\alpha}}$.

Proof. That $w(G)=\alpha$ is routine. The adjoint map

$$
\widehat{h}: \widehat{F^{\kappa}}=\bigoplus_{\kappa} \widehat{F} \rightarrow \widehat{G}=\bigoplus_{n<\omega}\left(\bigoplus_{\alpha_{n}} \mathbb{Z}\left(p_{n}\right)\right)
$$

is injective, so $r_{0}(\widehat{F})=0$ and there is $n<\omega$ such that $r_{p_{n}}(\widehat{F})>0$. Then

$$
\kappa \leq \kappa \cdot r_{p_{n}}(\widehat{F})=r_{p_{n}}\left(\bigoplus_{\kappa} \widehat{F}\right) \leq r_{p_{n}}(\widehat{G})=\alpha_{n}<2^{2^{\alpha_{n}}}=\alpha_{n+2}
$$

and hence $2^{2^{\kappa}} \leq 2^{2^{\alpha_{n+2}}}=\alpha_{n+4}<\alpha$, as asserted.

6.3. Remark. In [8, (4.10(d))] the authors construct, for each cardinal $\alpha \geq \omega$, a compact non-Abelian group $G(\alpha)$ with $w(G(\alpha))=\alpha$ which admits no homomorphism onto any group of the form $F_{0} \times F_{1}$ with $\left|F_{i}\right|>1$.

6.4. Discussion. Returning to Theorem 1.3 and its embracing paragraphs, we recall that it is unknown whether every pseudocompact Abelian group $G$ of uncountable weight admits a proper pseudocompact group refinement. We address this question in our work in progress 2], conjecturing there an affirmative answer. (See also in this connection the forthcoming work [17, which is a comprehensive survey of the literature on this question and on the related question of whether such groups $G$ necessarily admit a proper dense pseudocompact subgroup.) In the interest of completeness and with the encouragement of the referee, we pose in Question 6.5(b) another question, concerning which however we make no conjecture. Of course, a positive answer to Question 6.5(b) would yield a positive answer to Question 6.5(a).

6.5. Question. (a) Does every pseudocompact Abelian group of uncountable weight admit a proper pseudocompact group refinement?

(b) Does every pseudocompact Abelian group of uncountable weight admit a proper pseudocompact group refinement of maximal weight?

\section{REFERENCES}

[1] F. S. Cater, Paul Erdős, and Fred Galvin, On the density of $\lambda$-box products, General Topology and Its Applications 9 (1978), 307-312. MR 80e:54004

[2] W. W. Comfort and Jorge Galindo, Extremal pseudocompact topological groups (title tentative) (2000). Manuscript in preparation.

[3] W. W. Comfort and Dieter Remus, Imposing pseudocompact group topologies on Abelian groups, Fundamenta Math. 142 (1993), 221-240. MR 94g:22006

[4] W. W. Comfort and Dieter Remus, Abelian torsion groups with a pseudocompact group topology, Forum Mathematicum 6 (1994), 323-337. MR 95d:20096

[5] W. W. Comfort and Dieter Remus, Pseudocompact refinements of compact group topologies, Math. Zeitschrift 215 (1994), 337-346. MR 95f:54035

[6] W. W. Comfort and Lewis C. Robertson, Proper pseudocompact extensions of compact Abelian group topologies, Proc. Amer. Math. Soc. 86 (1982), 173-178. MR 83k:22011

[7] W. W. Comfort and Lewis C. Robertson, Cardinality constraints for pseudocompact and for totally dense subgroups of compact Abelian groups, Pacific J. Math. 119 (1985), 265-285. MR 87i:22002 
[8] W. W. Comfort and Lewis C. Robertson, Extremal phenomena in certain classes of totally bounded groups, Dissertationes Math. 272 (1988), 48 pages, Rozprawy Mat. Polish Scientific Publishers, Warszawa. MR 89i:22001

[9] W. W. Comfort and Kenneth A. Ross, Topologies induced by groups of characters, Fundamenta Math. 55 (1964), 283-291. MR 30:183

[10] W. W. Comfort and Kenneth A. Ross, Pseudocompactness and uniform continuity in topological groups, Pacific J. Math. 16 (1966), 483-496. MR 34:7699

[11] W. W. Comfort and Jan van Mill, Concerning connected, pseudocompact Abelian groups, Topology and Its Applications 33 (1989), 21-45. MR 90k:54002

[12] D. N. Dikranjan and D. B. Shakhmatov, Pseudocompact topologizations of groups, Zbornik radova Filozofskog fakulteta u Nišu Serija Matematika 4 (1990), 83-93. MR 92k:54043

[13] D. N. Dikranjan and D. B. Shakhmatov, Algebraic structure of the pseudocompact groups, 1991, Report 91-19, pp. 1-37. York University, Ontario, Canada.

[14] Dikran N. Dikranjan and Dmitri B. Shakhmatov, Algebraic structure of pseudocompact groups, Memoirs Amer. Math. Soc. 133, ix + 83 pages, 1998. MR 98j:22001

[15] E. K. van Douwen, The weight of a pseudocompact (homogeneous) space whose cardinality has countable cofinality, Proc. Amer. Math. Soc. 80 (1980), 678-682. MR 82a:54009

[16] László Fuchs, Infinite Abelian Groups, vol. I, Academic Press, New York-San FranciscoLondon, 1970. MR 41:333

[17] Jorge Galindo, Dense pseudocompact subgroups and finer pseudocompact group topologies, Sci. Math. Japan 55 (2001), 627-640.

[18] Edwin Hewitt, Rings of real-valued continuous functions I, Trans. Amer. Math. Soc. 64 (1948), 45-99. MR 10:126e

[19] Edwin Hewitt and Kenneth A. Ross, Abstract Harmonic Analysis, volume I, Die Grundlehren der mathematischen Wissenschaften in Einzeldarstellungen, volume 115, Springer Verlag, Berlin-Göttingen-Heidelberg, 1963. MR 28:158

[20] Edwin Hewitt and Kenneth A. Ross, Extensions of Haar measure and of harmonic analysis for locally compact Abelian groups, Math. Annalen 160 (1965), 171-194. MR 32:4208

[21] J. Mycielski, Some properties of connected compact groups, Colloq. Math. 5 (1958), 51-79. MR 20:6479

[22] André Weil, Sur les Espaces à Structure Uniforme et sur la Topologie Générale, Publ. Math. Univ. Strasbourg, vol. 551, Hermann and Cie, Paris, 1938.

Department of Mathematics, Wesleyan University, Middletown, Connecticut 06459

E-mail address: wcomfort@wesleyan.edu

Departamento de Matemáticas, Universitat Jaume I, 8029-AP Castellón, Spain

E-mail address: jgalindo@mat.uji.es 